\title{
Modulation of Phosphopeptide Fragmentation via Dual Spray Ion/Ion Reactions using a Sulfonate-Incorporating Reagent
}

\author{
Victoria C. Cotham, William M. McGee and Jennifer S. Brodbelt* \\ Department of Chemistry \\ The University of Texas at Austin \\ Austin, TX, USA 78712
}

Correspondence to: Jennifer Brodbelt, jbrodbelt@cm.utexas.edu

Supporting Information: Supplement figures include a CID product ion spectrum of unmodified KKALRRQEpTVDAL 2+, representative ESI-MS spectra before and after ion/ion reactions with FBDSA, an MS2 spectrum demonstrating covalent conversion of a phosphopeptide to its FBDSAmodified analogue, MS3 CID product ion spectrum for a Schiff base modified peptide, reaction schemes for N-terminal modification of peptides via carbamylation and SPITC derivatization, ESIMS spectra for a carbamylated and SPITC modified phosphopeptide, and a CID product ion spectrum for a SPITC modified phosphopeptide. 
Figure S1. Summary of phosphopeptide cation derivatization with FBDSA anions via front-end ion/ion-mediated bioconjugation.
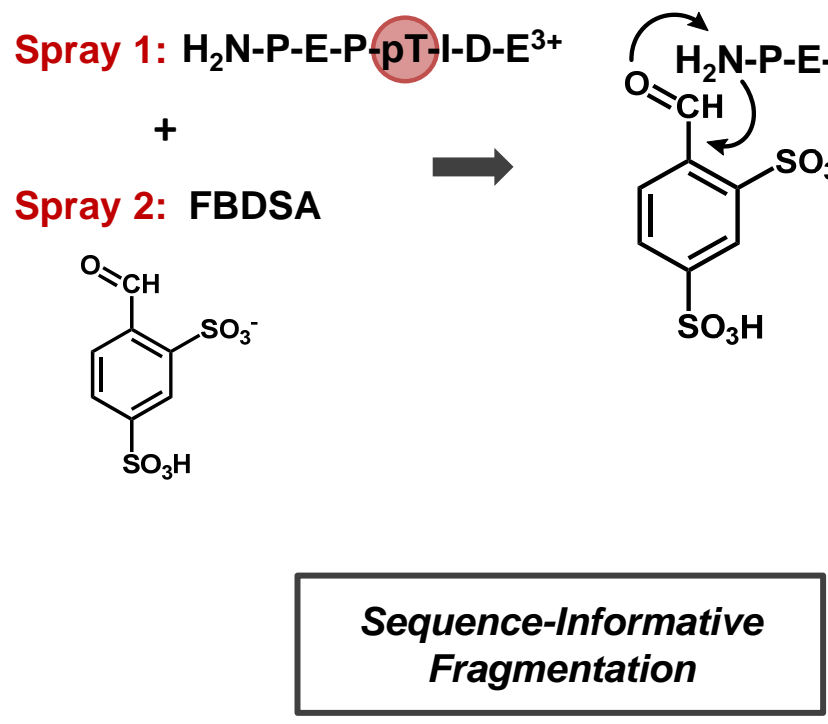
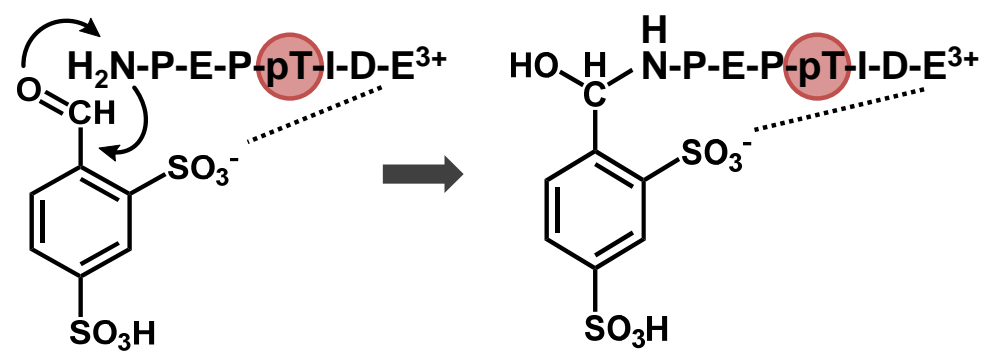

$\mathrm{MS}^{2}-\mathrm{H}_{2} \mathrm{O}$

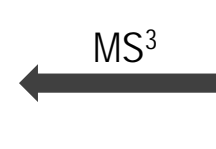

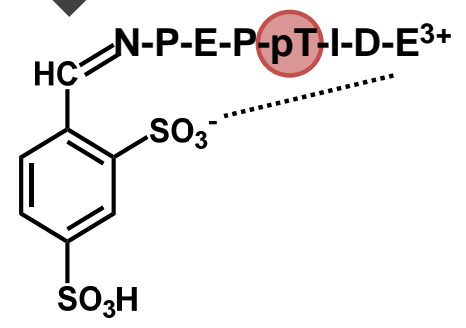


Figure S2. CID product ion spectrum of the 2+ charge state of KKALRRQEpTVDAL. Neutral loss of phosphate is indicated by "-P" in the product ion label.

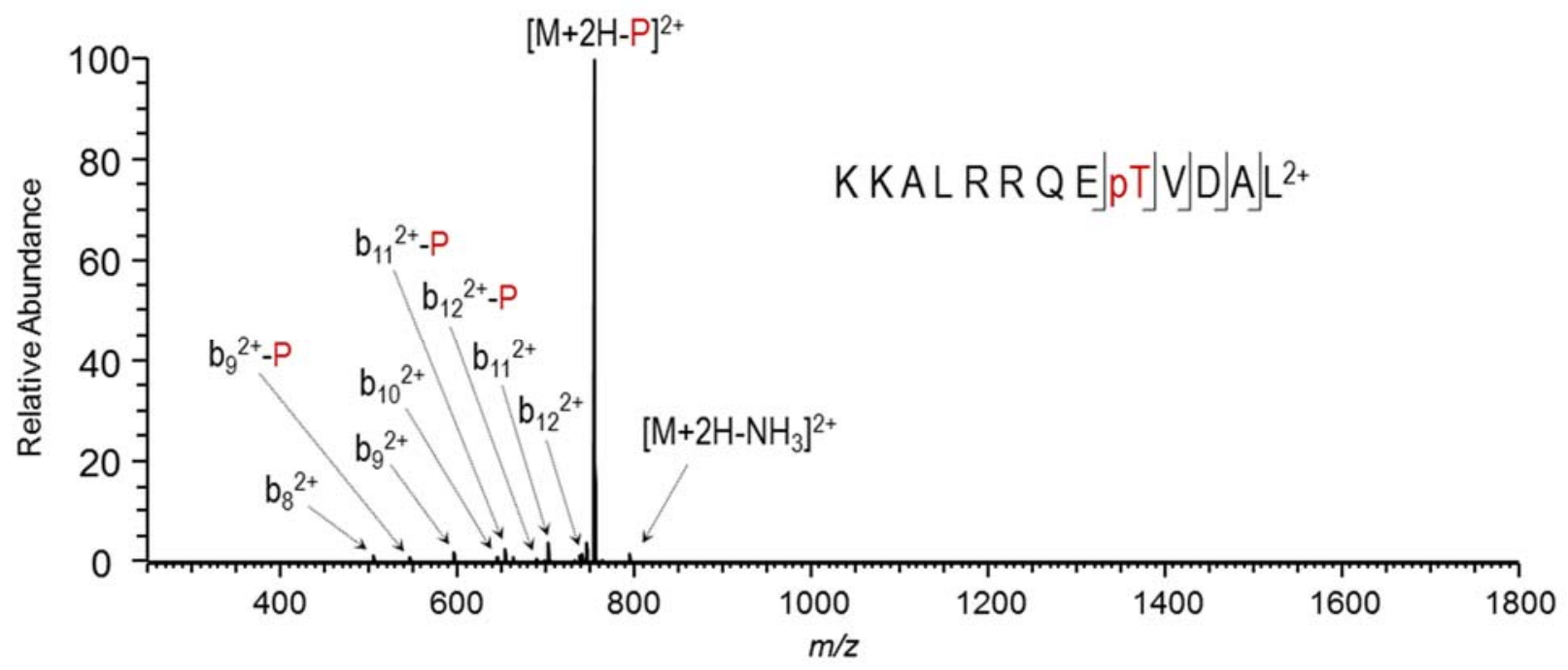


Figure S3. Process for online modification of phosphopeptides with FBDSA using a dual spray reactor. ESI spectra for (a) unreacted and (b) dual source reacted KKALRRQEpTVDAL. Chargereduced electrostatic complexes are formed at atmospheric pressure between multiply charges phosphopeptide cations and FBDSA reagent anions (denoted by the " $\Delta$ " subscript), and transferred and mass analyzed in the linear ion trap. (c) Low-energy collisional activation of these ion/ion intermediates promotes concomitant imine bond formation and dehydration to form a covalent Schiff base product ( $\downarrow)$. (d) CID of the resulting Schiff base phosphopeptide results in sequenceinformative fragmentation.

(a) MS 1 : KKALRRQEpTVDAL

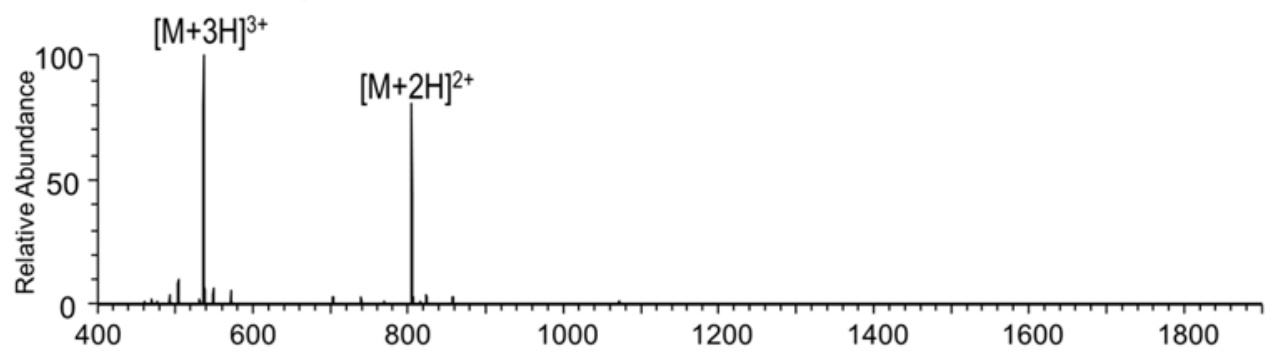

(b) MS'1: KKALRRQEpTVDAL + FBDSA

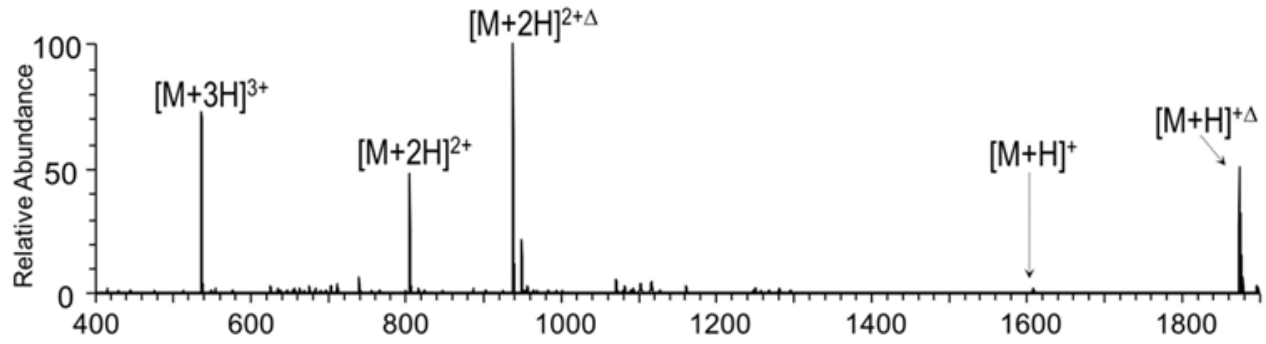

(c) MS 2 : KKALRRQEpTVDAL ${ }^{2+\Delta}$

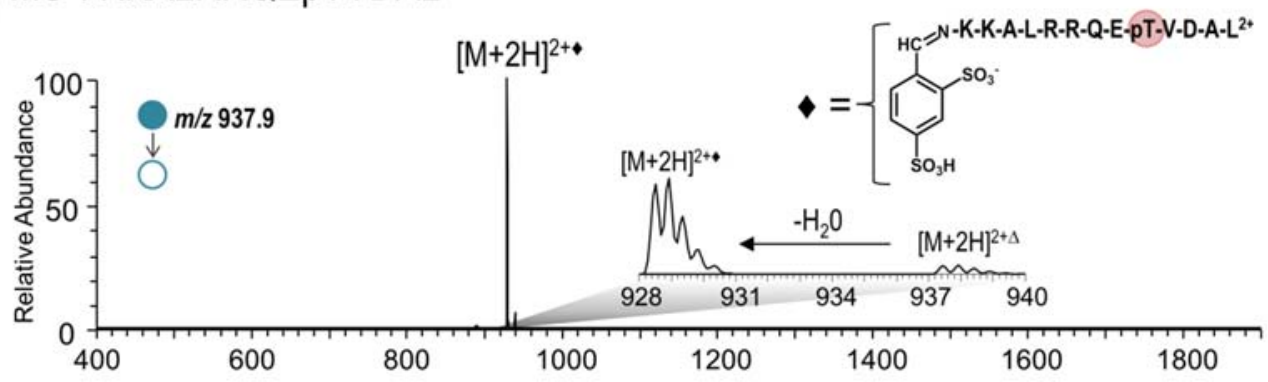

(d) MS 3 : KKALRRQEpTVDAL ${ }^{2+\bullet}$

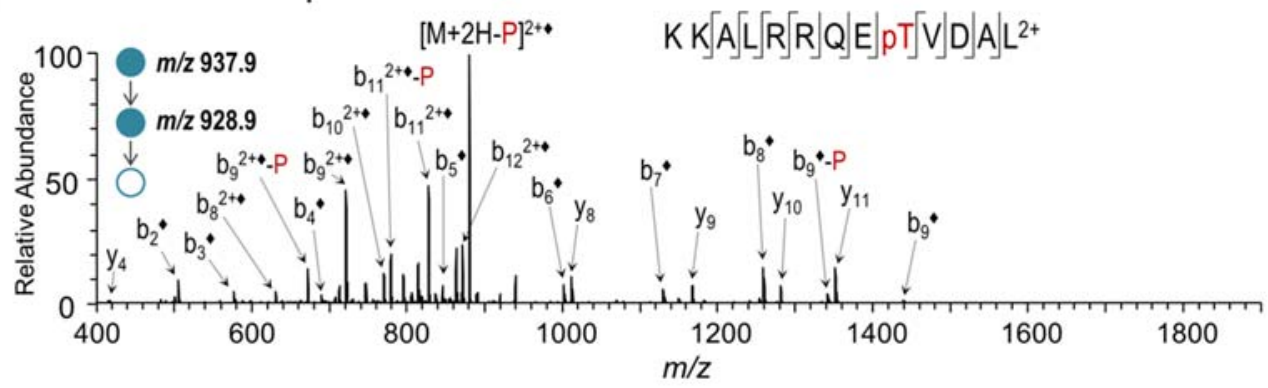


Figure S4. (a) Dual source mass spectrum of tryptic peptide LIEDAEpYAAR. Electrostatic FBDSA/phosphopeptide complex formation is denoted by the " $\Delta$ " subscript. Comparative CID product ion mass spectra of LIEDAEpYAAR $(1+)$ before and after Schiff base modification are shown in (b) and (c), where: (b) $\mathrm{MS}^{2}$ CID mass spectrum of unlabeled peptide and (b) $\mathrm{MS}^{3}$ CID mass spectrum following online dual spray reactor-initiated derivatization. The addition of " the label indicates covalent FBDSA Schiff base modification, "-P" denotes loss of phosphate, and "o" indicates neutral loss of water or ammonia.

(a) MS' ${ }^{1}$ : LIEDAEpYAAR + FBDSA

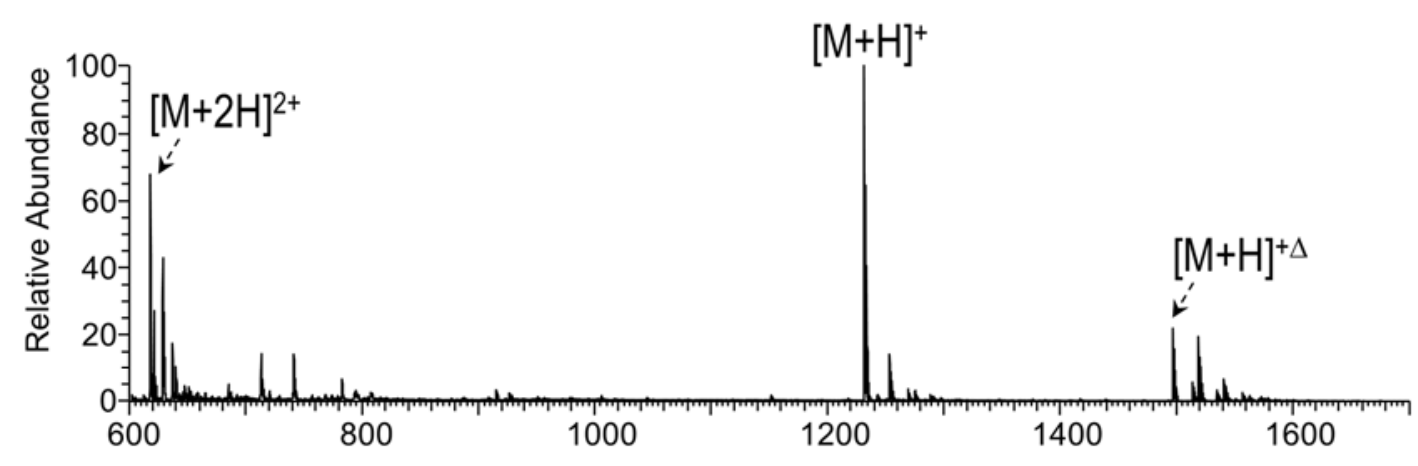

(b) Unmodified: LI|E[D[A]E[pYAAR+

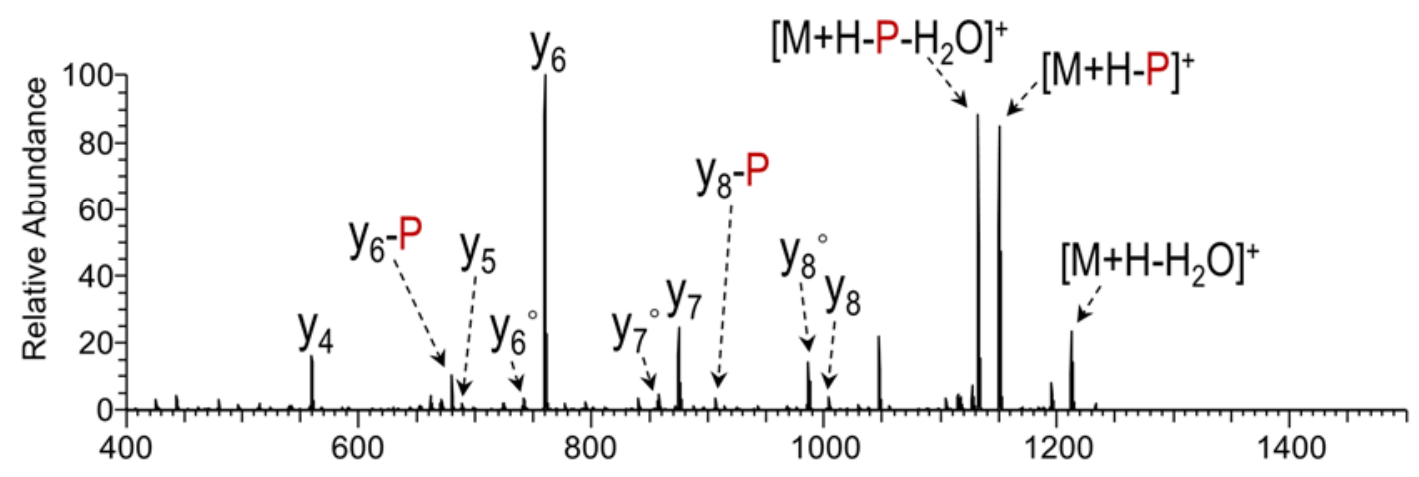

(c) Schiff Base: LI|E[D[A]E[pYAAR+

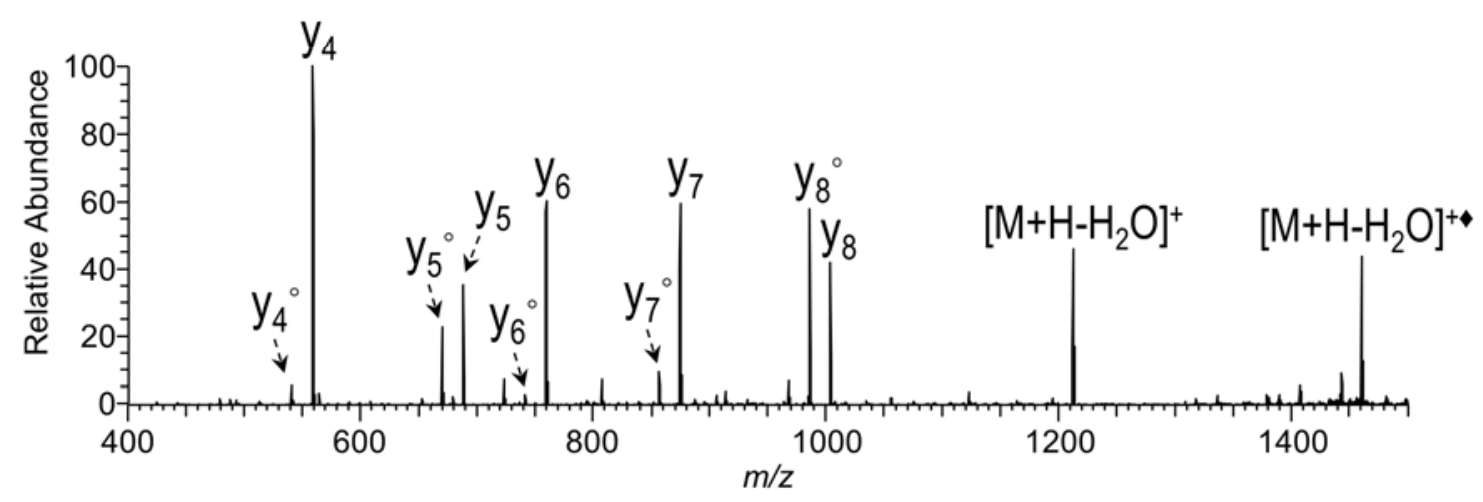


Figure S5. (a) Dual source mass spectrum of peptide LHpSPQSLPR. Electrostatic FBDSA/phosphopeptide complex formation is denoted by the " $\Delta$ " subscript. Comparative CID product ion mass spectra of LHpSPQSLPR (1+) before and after Schiff base modification are shown in (b) and (c), where: (b) $\mathrm{MS}^{2}$ CID mass spectrum of unlabeled peptide and (b) $\mathrm{MS}^{3} \mathrm{CID}$ mass spectrum following online dual spray reactor-initiated derivatization. The addition of " the label indicates covalent FBDSA Schiff base modification, "-P" denotes loss of phosphate, and "o" indicates neutral loss of water or ammonia.

(a) $\mathrm{MS}^{1}:$ LHpSPQSLPR + FBDSA

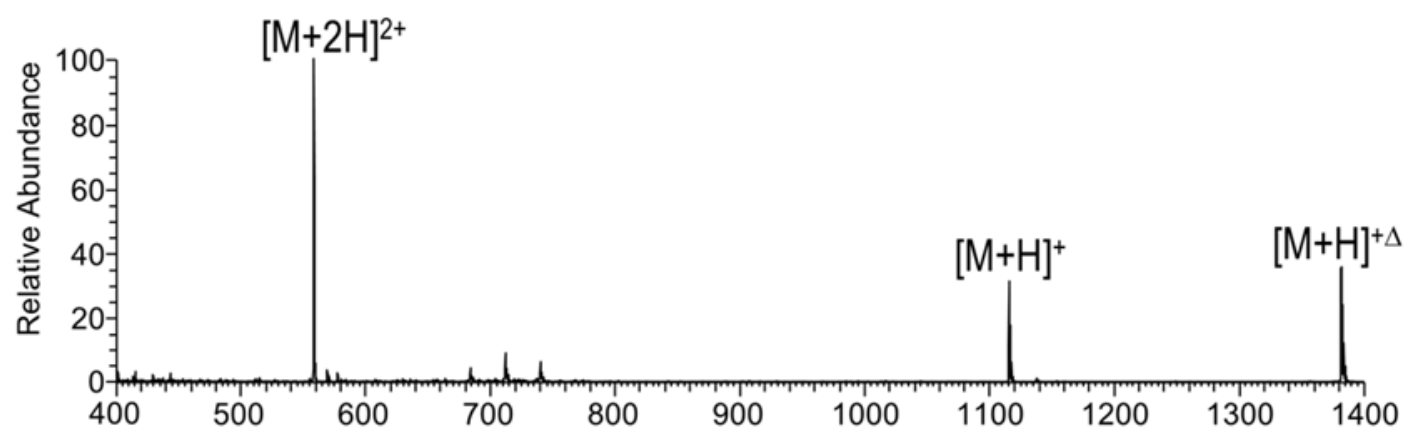

(b) Unmodified: $\mathrm{LH}\left\lceil\mathrm{pS} \sqrt[\mathrm{P} Q]{\mathrm{S}} \sqrt{\mathrm{L}} \mathrm{P} \mathrm{R}^{+}\right.$

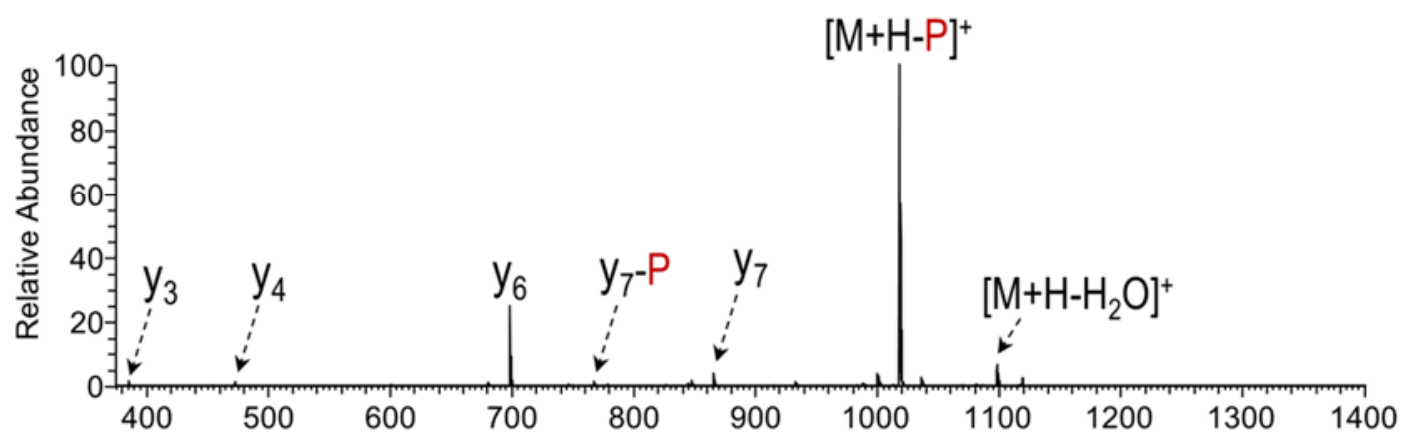

(c) Schiff Base: $\mathrm{L}\left[\mathrm{H} / \mathrm{pS} / \mathrm{P}\left[\mathrm{Q}\left[\mathrm{S}\left[\mathrm{L} P \mathrm{R}^{+}\right.\right.\right.\right.$

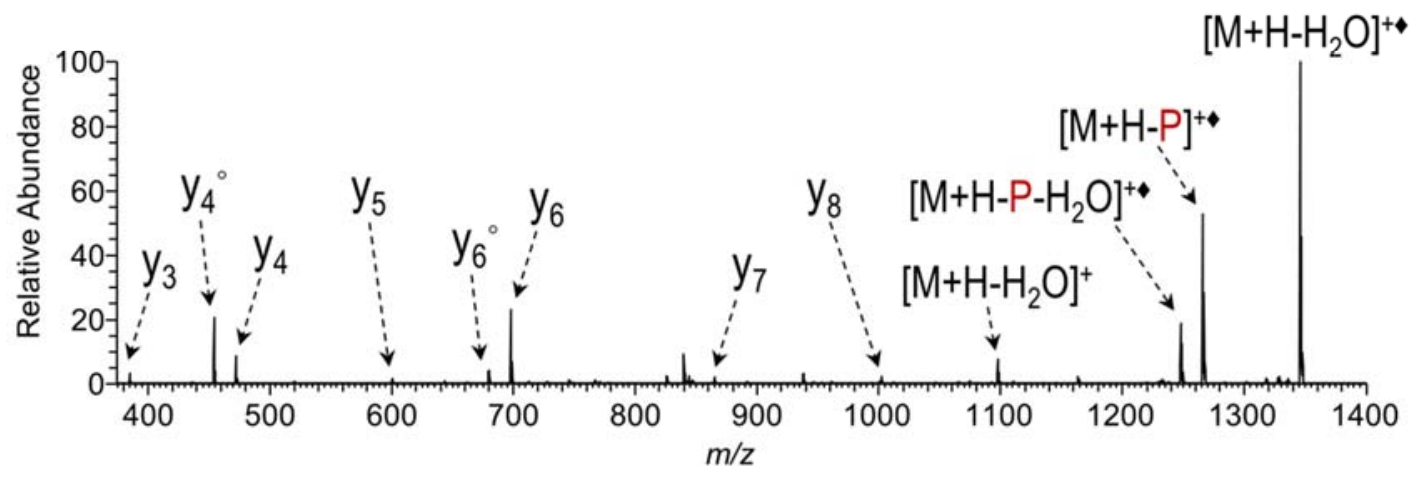


Figure S6. Reaction scheme for N-terminal (a) carbamylation, and (b) 4-sulfophenyl isothiocyanate (SPITC) derivatization of a peptide.

(a) Carbamylation
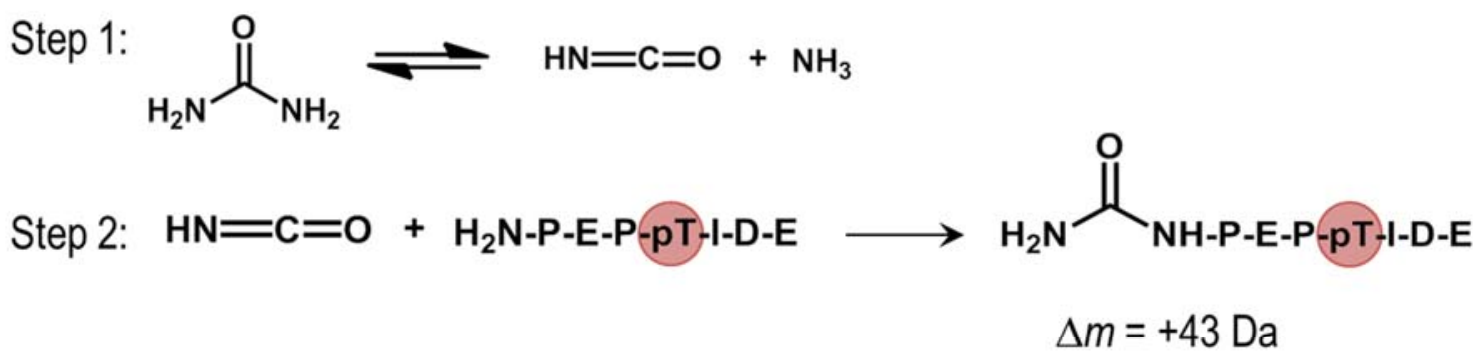

(b) SPITC Derivatization

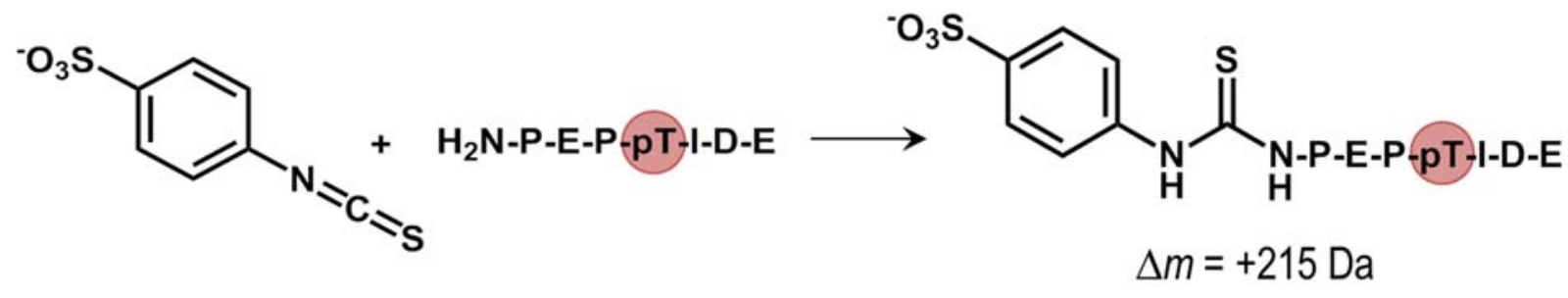


Figure S7. Mass spectra of RQpSVELHSPQSLPR prior to (a) and after (b) carbamylation, (c) SPITC derivatization, and (d) ion/ion reaction with FBDSA.

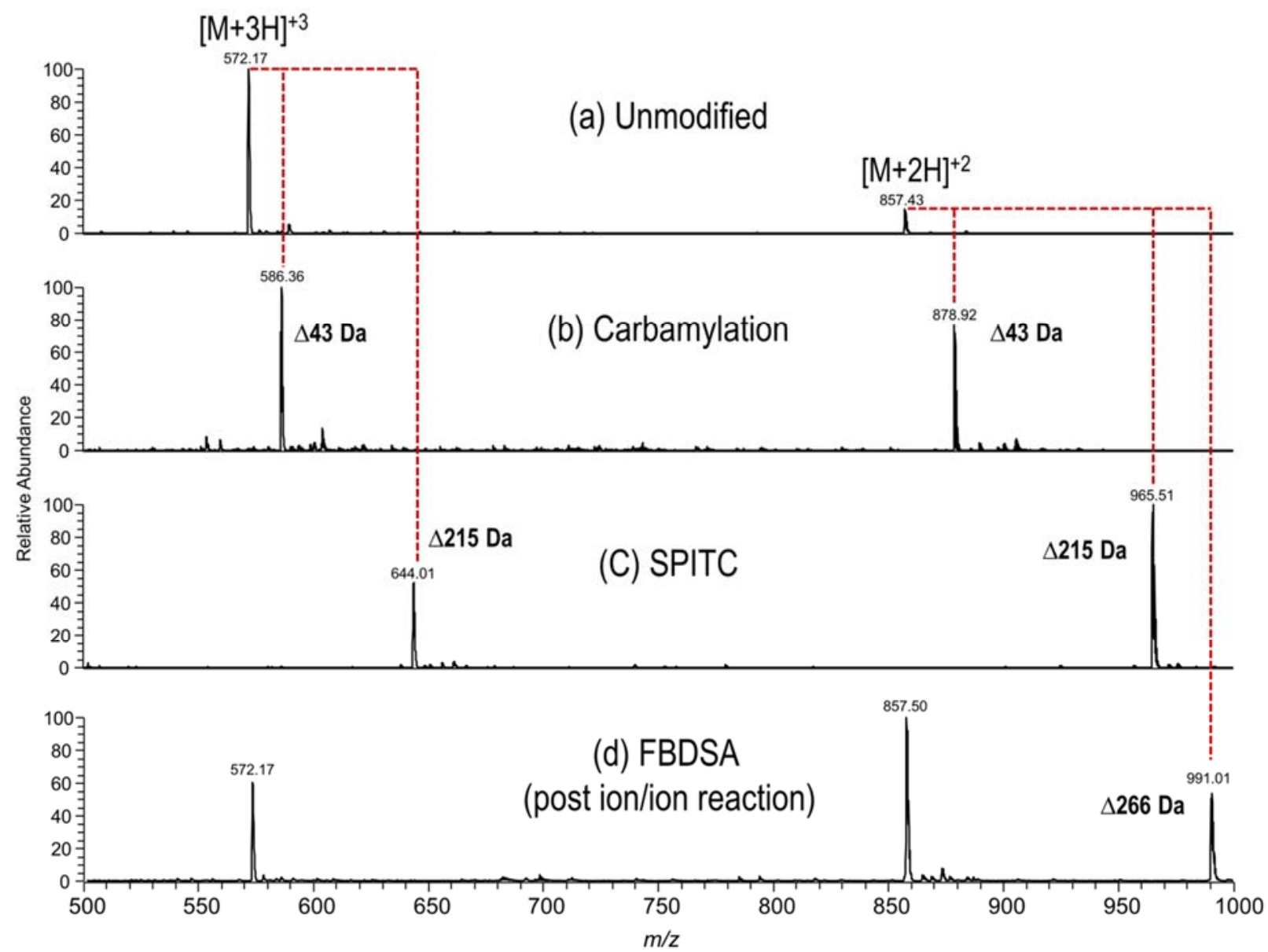


Figure S8. CID product ion spectrum of SPITC modified RQpSVELHSPQSLPR 2+. The abundant product ion a $\mathrm{m} / \mathrm{z} 878$ corresponds to sulfanilic acid cleavage from the SPITC tag. A modified $b_{1}$ ion results from the Edmund degradation process shown in the inset.

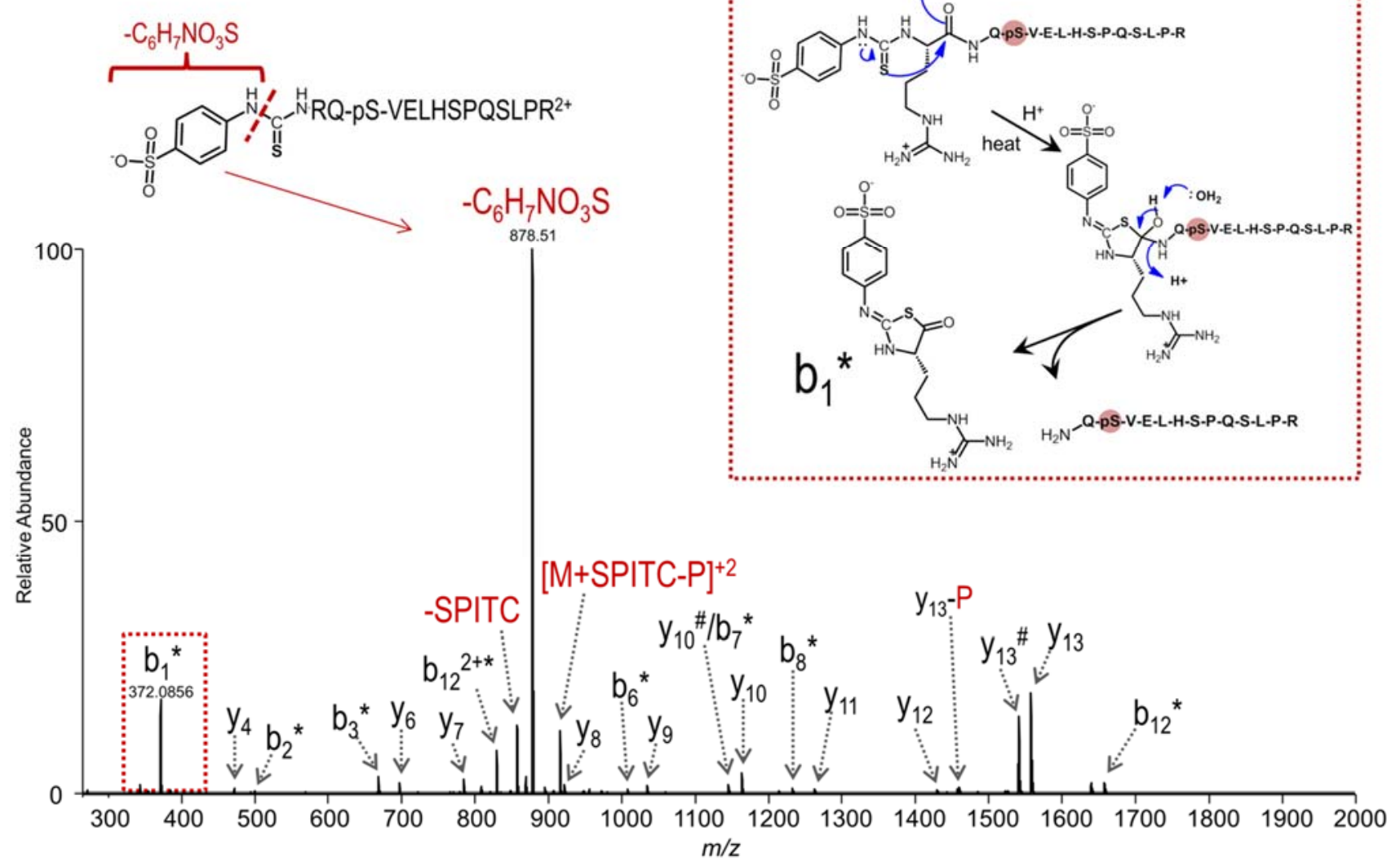

